\title{
Ventricular Fibrillation Due to Early Repolarization Syndrome in the Wake of Hypothermia Due to Fulminant Acute Disseminated Encephalomyelitis
}

Kyoichiro Yazaki, MD; Yoichi Ajiro, MD, PhD; Kunihiko Shimizu, MD;

Fumiaki Mori, MD, PhD; Nobue Yagihara, MD; Akinori Sato, MD, PhD;

Tatsuya Takahashi, MD, PhD; Kazunori Iwade, MD, PhD
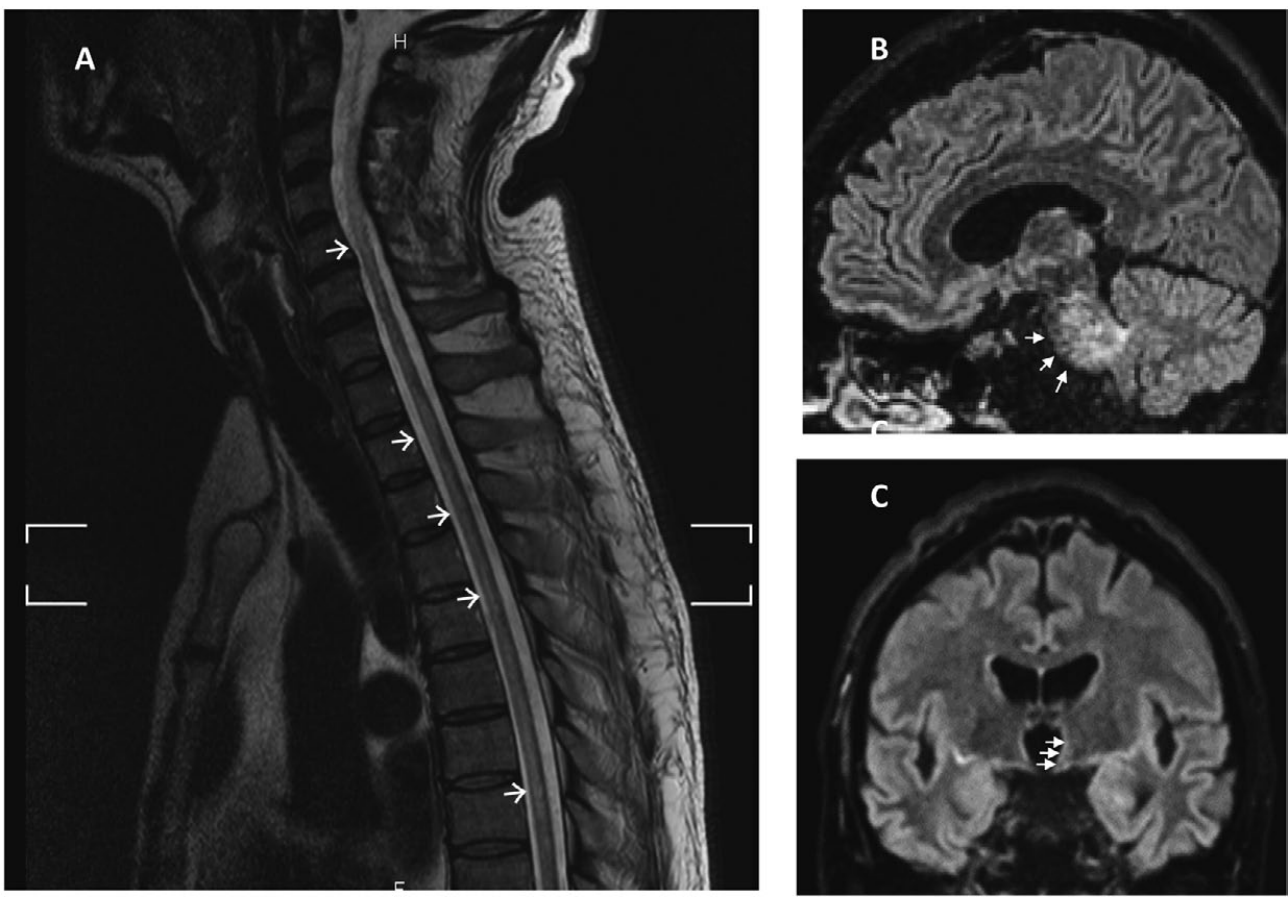

Figure 1. (A,B) Sagittal T2-weighted magnetic resonance imaging (MRI) showing a broad high-intensity area in the (A) spinal cord and (B) pons (white arrows). (C) The high-intensity lesion was also observed at the edge and near the hypothalamus on fluid-attenuated inversion recovery MRI, coronal view (white arrows).

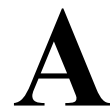
44-year-old man was hospitalized for meningitis, with increased cell count and protein level in the cerebrospinal fluid (CSF). Despite treatment with antibiotics, he developed disturbed consciousness after 8 days. A diagnosis of acute disseminated encephalomyelitis
(ADEM) was made based on elevated oligoclonal IgG bands, myelin basic protein level, and $80 \%$ monocytes in the CSF with absence of serum antibodies against commonly causal viruses. Magnetic resonance imaging (MRI) indicated extensive damage to the brainstem and cerebellum,

Received July 9, 2017; revised manuscript received August 2, 2017; accepted August 21, 2017; released online September 20,2017 Time for primary review: 23 days

Department of Cardiology (K.Y., Y.A., K.S., F.M., K.I.), Department of Neurology (T.T.), National Hospital Organization Yokohama Medical Center, Yokohama; Department of Cardiology, Tokyo Women's Medical University, Tokyo (Y.A., F.M.); and Department of Cardiovascular Biology and Medicine, Niigata University Graduate School of Medical and Dental Sciences, Niigata (N.Y., A.S.), Japan

Mailing address: Kyoichiro Yazaki, MD, Department of Cardiology, National Hospital Organization Yokohama Medical Center, 3-60-2 Harajuku, Totsuka-ku, Yokohama 245-8575, Japan. E-mail: kamisamakaranookurimono@gmail.com

ISSN-1346-9843 All rights are reserved to the Japanese Circulation Society. For permissions, please e-mail: cj@j-circ.or.jp 
A

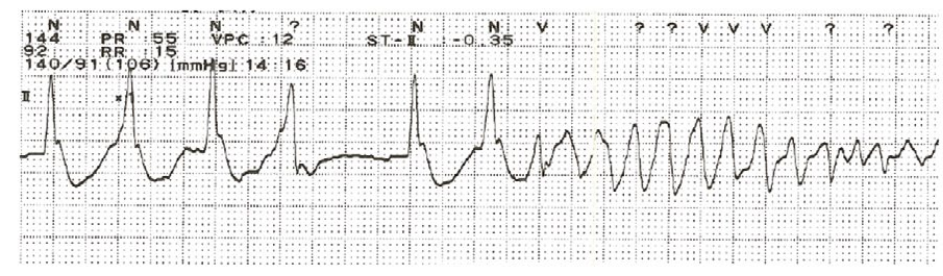

B

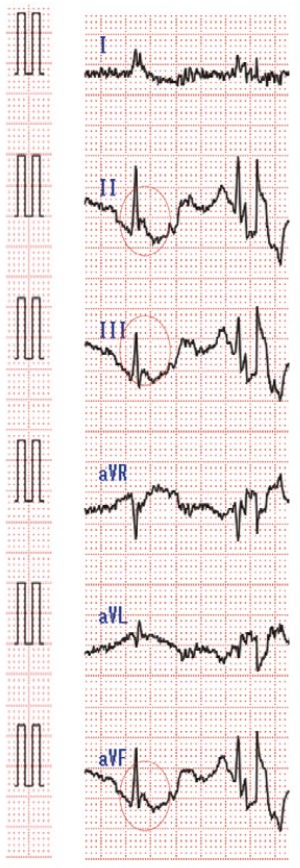

$\mathrm{BT}: 32.9^{\circ} \mathrm{C}$
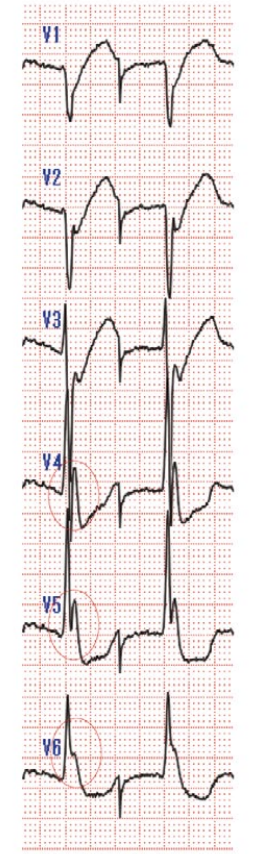

C

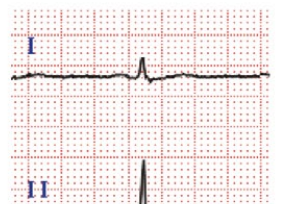

$\mathrm{BT}: 36.5^{\circ} \mathrm{C}$

Figure 2. (A) Electrocardiogram (ECG) showing polymorphic ventricular tachycardia triggered by frequent premature ventricular contractions. (B) Prominent J wave was observed in the inferolateral leads (red dotted circle) on 12-lead ECG immediately after the termination of ventricular fibrillation. (C) Following normalization of the body temperature and acid-base equilibrium, the $\mathrm{J}$ wave was no longer visible on ECG. BT, body temperature.

including the edges of and regions near the hypothalamus (Figure 1). Three months later, his body temperature decreased to $34^{\circ} \mathrm{C}$, but he felt hot and had sudoresis. Suddenly, repetitive ventricular fibrillation (VF) after prominent J wave developed (Figure 2A). On emergency cardiac catheterization and transthoracic echocardiography, no signs of structural heart disease were seen. Notably, his temperature decreased to $32.9^{\circ} \mathrm{C}$ while the electrocardiogram (ECG) showed prominent $\mathbf{J}$ wave in II, III, aVF, and V4-V6 (Figure 2B). He was rewarmed during deep sedation to inhibit repetitive $\mathrm{VF}$, and no $\mathrm{J}$ wave was observed after the temperature normalized to $36.5^{\circ} \mathrm{C}$ (Figure 2C). Nextgeneration gene sequencing (HiSeq2000 ${ }^{\mathrm{TM}}$; Illumina, San Diego, CA, USA) screening for 580 mutations and 20 microRNAs detected no mutation except the NDRG4 variant seen in $0.05 \%$ of the general population. VF did not recur for 3 years by avoiding hypothermia.

This case demonstrates that ADEM with hypothermia due to thermoregulatory disorder can cause VF associated with early repolarization syndrome (ERS). ADEM is an immune-mediated demyelinating condition triggered by an inflammatory response to virus, vaccine, or other infectious agent. ${ }^{1}$ It predominantly affects the white matter of the brain and spinal cord, occasionally involving the brainstem, diencephalon, or hypothalamus. Such systemic neural damage is reported to lead to functional disorders. ${ }^{2}$ In this case, a thermoregulatory disorder was suspected because the patient complained of heat and sudoresis. Together with the T2-weighted MRI findings, ADEM-mediated hypothalamus damage would cause thermoregulatory disorder.

Early repolarization, characterized by $\mathbf{J}$ wave or slur after the QRS complex on ECG, is often seen in young healthy people. Some patients with idiopathic VF associated with ERS also have an augmented $\mathbf{J}$ wave before VF develops. Both repolarization and depolarization abnormalities can cause ERS, and the augmented cardiac transmural dispersion and accompanying phase-2 re-entry can provoke VF, based on genetic abnormality related to cardiac ion currents. ${ }^{3,4}$ Unusual physiological conditions such as hypothermia can also cause the early repolarization known as Osborn $\mathbf{J}$ wave, which is augmented with the severity of hypothermia. ${ }^{5}$ Interestingly, hypothermia affects 
the transient outward potassium current, resulting in an accelerated epicardial action potential notch. ${ }^{6}$ Although no evidence for correlation was seen on genetic analysis, hypothermia would have played at least some role in this case.

To our knowledge, this is the first report of hypothermia triggering ERS and subsequent VF after ADEM-mediated hypothalamus disorder.

\section{Acknowledgments}

We thank Editage (www.editage.jp) for English-language editing.

\section{Funding Source}

This case report was funded by the Clinical Research Division of National Hospital Organization Yokohama Medical Center. The next-generation gene sequencing was conducted using funds of Niigata University.

\section{Disclosures}

The authors declare no conflicts of interest.

\section{References}

1. Garg RK. Acute disseminated encephalomyelitis. Postgrad Med $J$ 2003; 79: 11-17.

2. Benarroch EE. The central autonomic network: Functional organization, dysfunction, and perspective. Mayo Clin Proc 1993; 68: $988-1001$.

3. Obeyesekere MN, Klein GJ, Nattel S, Leong-Sit P, Gula LJ, Skanes AC, et al. A clinical approach to early repolarization. Circulation 2013; 127: 1620-1629.

4. Antzelevitch C, Yan GX, Ackerman MJ, Borggrefe M, Corrado $\mathrm{D}$, Guo J, et al. J-wave syndromes expert consensus conference report: Emerging concepts and gaps in knowledge. J Arrhythm 2016; 32: 315-339.

5. Higuchi S, Takahashi T, Kabeya Y, Hasegawa T, Nakagawa S, Mitamura H. J waves in accidental hypothermia. Circ J 2014; 78: $128-134$.

6. Chhabra L, Devadoss R, Liti B, Spodick DH. Electrocardiographic changes in hypothermia: A review. Ther Hypothermia Temp Manag 2013; 3: 54-62. 